\title{
Photonic Bands in Patterned Silicon-on-Insulator Waveguides
}

\author{
M. Patrini, M. Galli, F. Marabelli, M. Agio, L. C. Andreani, D. Peyrade, and Yong Chen
}

\begin{abstract}
One- and two-dimensional photonic crystals are obtained by electron beam lithography and reactive ion etching on silicon-on-insulator waveguides. Variable-angle reflectance is measured and calculated in the 0.2-2-eV energy range, giving evidence of the quasi-guided modes of the photonic structure. Photonic bands in the one-dimensional case are determined and compare very well to those calculated with an expansion on the basis of the waveguide modes.
\end{abstract}

Index Terms-Modeling, optical materials, reflectometry, waveguides.

\section{INTRODUCTION}

$\mathbf{T}$ HE BEHAVIOR of photonic crystals embedded in planar waveguides is a key element to be understood for the realization of optical functionalities in photonic integrated circuits (PICs). The ideal one-dimensional (1-D) and two-dimensional (2-D) lattice cases are now well understood, but the optical properties of a thin-layer photonic crystal inserted into a waveguide are still not completely known and are currently of great interest [1]-[20]. In particular, a precise knowledge of the photonic bands is essential for any application.

In this work, we investigate the photonic band structure of photonic crystals fabricated by electron beam lithography and reactive ion etching techniques on silicon-on-insulator (SOI) waveguides. The choice of the material and the spectral range under study (around $1.55 \mu \mathrm{m}$ ) are motivated by the telecom application possibilities. The resulting samples consist of 1-D (stripes) and 2-D (triangular and graphite) lattices of different periodicities lying on silicon dioxide. Here we focus on two 1-D structures with different air fractions and discuss how the different optical behavior of the samples may be related to the topical issue of diffraction losses of quasi-guided modes lying above the light line.

Our measurements are based on the surface coupling technique proposed by Astratov et al. [8], [9], [11] on III-V photonic crystals. The method relies on the observation of sharp resonances in reflectance $(R)$ spectra of collimated light incident on the surface of the photonic crystal. We measure the polarized specular $R$ spectra of the samples for an incidence angle varying from near-normal incidence to $70^{\circ}$. Resonant structures due to the coupling of the external radiation to the eigenmodes

Manuscript received August 31, 2001; revised March 1, 2002.

M. Patrini, M. Galli, F. Marabelli, M. Agio, and L. C. Andreani are with the INFM and Dipartimento di Fisica “A. Volta," Università degli Studi di Pavia, I27100 Pavia, Italy (e-mail: patrini@ fisicavolta.unipv.it).

D. Peyrade and Y. Chen are with the Laboratoire de Photonique et de Nanostructures, LPN-CNRS, 91460 Marcoussis, France (e-mail yong.chen@lpn.cnrs.fr).

Publisher Item Identifier S 0018-9197(02)05691-9. of the photonic crystals are observed, together with interference oscillations due to the SOI layered structure. We identify several resonances in the polarized optical spectra and we trace their energy dependence on the incidence angle. In this way, we are able to map the bands of these photonic crystals above the light line in the reduced Brillouin zone.

The experimental results are compared with two complementary theoretical treatments. The photonic bands of the waveguide-embedded photonic crystal are calculated by a newly developed method, which consists of expanding the electromagnetic field on the basis of waveguide modes coupled by the off-diagonal terms of the dielectric tensor, where the waveguide is defined to have an average dielectric constant. This method yields not only the photonic modes below the light line [6], which are truly guided modes and have no diffraction losses, but also the quasi-guided modes (or "guided resonances") above the light line which are probed by the external field in a surface reflectance experiment. The reflectance of the patterned multilayer structure is also calculated by the scattering matrix method developed by Whittaker and Culshaw [10]. The resonant structures of the $R$ spectra correspond to the experimentally measured ones and to the calculated energies of the photonic bands in the radiative region.

\section{EXPERIMENTAL DETAILS}

The photonic waveguides investigated in this work are obtained by standard electron beam lithography and reactive ion etching techniques on Smart Cut SOI wafers by SOITEC. The thicknesses of the silicon and insulator layers were $0.26 \mu \mathrm{m}$ and $1 \mu \mathrm{m}$, respectively. First, the electron beam patterning is performed by a JEOL 5D2U vector Scan generator operating at an energy of $50 \mathrm{keV}$ on a single layer of polymethylmetacrylate (PMMA) resist (950 K molecular weight and $150 \mathrm{~nm}$ thickness). After development of the PMMA, a thin nickel layer is evaporated onto the silicon surface and lifted off by dissolution of the PMMA. The reactive ion etching of the Si layer is then performed in a Nextral NE110 system. The etching process uses an $\mathrm{SF}_{6} / \mathrm{CHF}_{3}$ gas mixture at a pressure of $10 \mathrm{mT}$ and an RF power of $15 \mathrm{~W}$. The etching rate is typically $50 \mathrm{~nm} / \mathrm{min}$ and reactive ion etching parameters are optimized for steeper sidewalls. Finally, the remaining Ni mask is removed in a nitric acid solution.

Several photonic crystals were prepared in this way, including 1-D lattices of stripes, 2-D graphite lattices of pillars, and triangular lattices of holes. In the following we focus our attention on two different 1-D structures: the first one with lattice constant $a=1 \mu \mathrm{m}$ and 0.7 air fraction (referred to hereafter as L3) and the second one with lattice constant $a=0.65 \mu \mathrm{m}$ and 0.18 

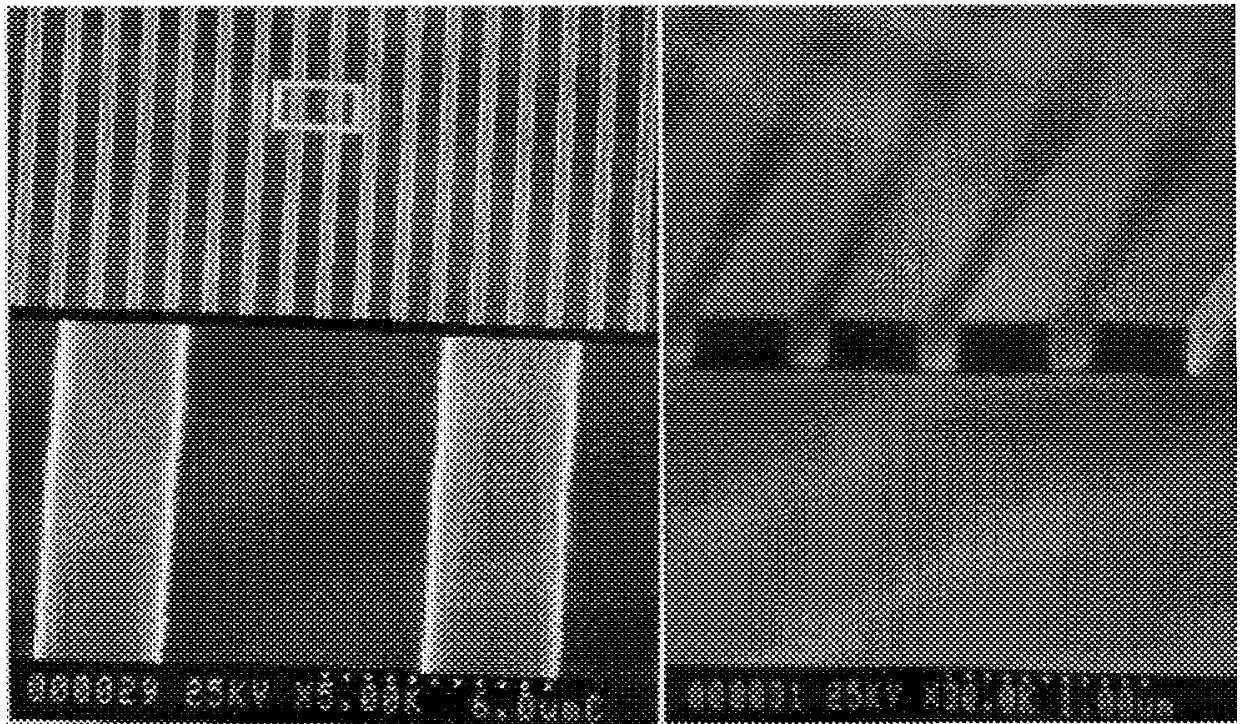

Fig. 1. SEMs of sample L3 (top view, left; scale refers to upper panel) and L4 (side view, right).

air fraction (L4). Scanning electron microscope (SEM) images of the two lattices are shown in Fig. 1 and show the excellent quality of the structures.

Spectroscopic ellipsometry measurements are used in a complementary way to characterize the quality and the thickness of the waveguides before patterning.

Variable angle specular reflectance is measured in the spectral range $0.25-2 \mathrm{eV}$ with a Fourier-transform spectrometer (Bruker IFS66) at a spectral resolution of $1 \mathrm{meV}$. The plane of incidence is perpendicular to the sample surface and the angle of incidence $\theta$ of the collimated beam from the spectrometer varies over the range $5^{\circ}-75^{\circ}$ with a step of $2.5^{\circ}$ and with angular resolution of $\pm 1^{\circ}$. A liquid-nitrogen-cooled InSb photodiode is used as the detector and a silver mirror is the absolute reflectance reference. Measurements were done for light incident along the $\Gamma-X$ orientation of the 1-D crystal (i.e., perpendicular to the stripes) both in transverse electric (TE) and transverse magnetic (TM) polarizations by means of a calcite Glan-Taylor polarizer.

\section{THEORETICAL SCHEME}

Two complementary theoretical approaches are adopted. In the first one, the photonic bands of the patterned waveguide at the parallel Bloch vector $k$ are calculated by expanding the magnetic field as

$$
\mathbf{H}_{\mathbf{k}}(\mathbf{r})=\sum_{\mathbf{G} \alpha} c_{\mathbf{k}+\mathbf{G}, \alpha} e^{i(\mathbf{k}+\mathbf{G}) \cdot \mathbf{r}} \mathbf{h}_{\mathbf{k}+\mathbf{G}, \alpha}(z)
$$

where $\mathbf{h}_{\mathbf{k}+\mathbf{G}, \alpha}(z), \alpha=1,2, \ldots$ are the guided modes of the air $/ \mathrm{Si} / \mathrm{SiO}_{2}$ waveguide at wavevector $\mathbf{k}+\mathbf{G}$ and the waveguide core made of patterned silicon is taken to have an average dielectric constant. The second-order equation for the magnetic field is transformed into a linear eigenvalue problem for the expansion coefficients $c_{\mathbf{k}+\mathbf{G}, \alpha}$, with the waveguide modes being coupled by the off-diagonal components of the inverse dielectric tensor. Just like in the case without a waveguide, a finite cutoff must be imposed for the reciprocal lattice vectors $\mathbf{G}$ and Ho's method of inverting the dielectric matrix has to be used for faster convergence [21]. This approach yields the energy dispersion of the guided modes whose energies lie below the light lines of the upper and lower claddings, as well as of the quasi-guided modes which lie above the two light lines when folded in the first Brillouin zone. It neglects coupling of the latter states to the leaky modes of the waveguide; therefore, it does not yield the imaginary part of the energy that corresponds to intrinsic radiation losses (out-of-plane diffraction in a transmission experiment). The resonance energies of quasi-guided modes are of central interest for the interpretation of the present experiments, since they correspond to the spectral positions of the resonant structures in reflectance, which arise when a quasi-guided mode is excited by the incoming beam.

In the second approach, we calculate the reflectance and transmittance (and also the diffraction) of the layered system by a plane-wave expansion in each layer, which yields the complex photonic bands at a given in-plane wavevector $\mathrm{k}$ and frequency $\omega$, and by propagating the field along the multilayer via a scattering matrix [10]. In the present case, we have four layers: air, patterned silicon, unpatterned $\mathrm{SiO}_{2}$, and the $\mathrm{Si}$ substrate. The frequency-dependent complex dielectric constants of $\mathrm{Si}$ and $\mathrm{SiO}_{2}$ are used in the calculation [22]. This numerical method is an exact solution of Maxwell's equations and contains all radiative effects in the structure.

For a parallel wavevector $\mathbf{k}$ along a symmetry direction of the lattice (in the 1-D case, the $\Gamma-X$ direction), specular reflection with respect to a vertical plane $(\mathrm{k}, z)$ is a symmetry operation and the TE or TM polarization of the incident wave is mantained in reflectance. Correspondingly, the photonic bands can be classified as even or odd with respect to vertical mirror symmetry. They are probed by a polarized incident wave as follows: a TM-polarized wave couples to even bands and a TE-polarized wave couples to odd bands. Note that for the present asymmetric SOI structure there is no mirror symmetry with respect to the waveguide plane (unlike in the ideal 1-D or 2-D case). 


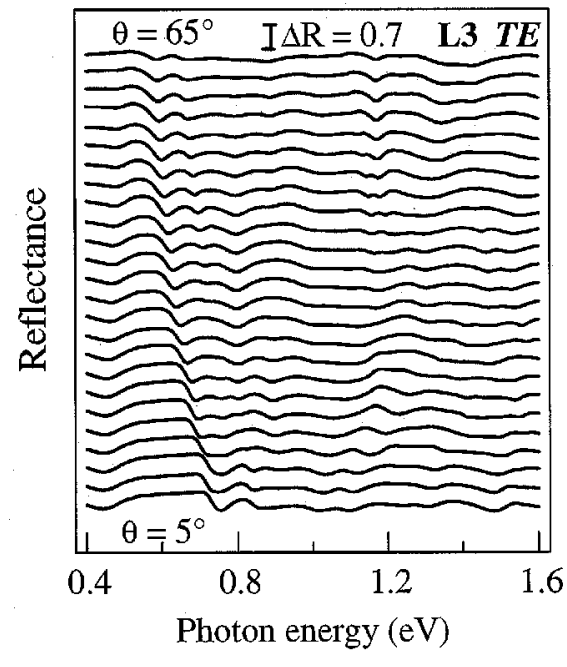

(a)

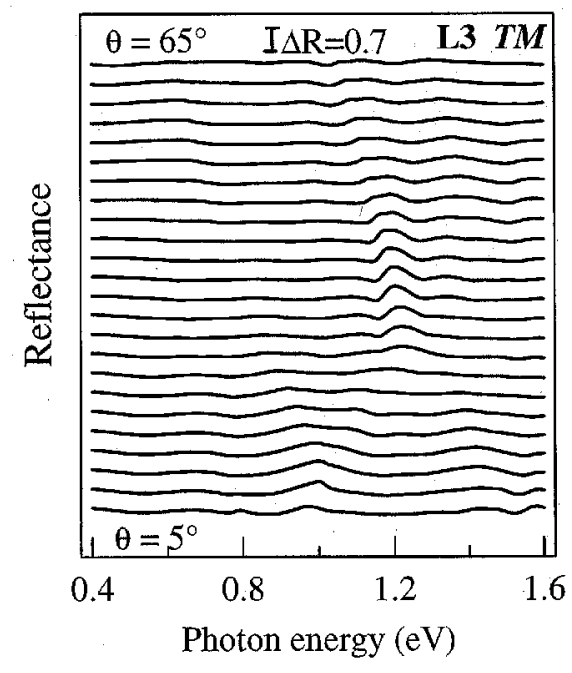

(b)

Fig. 2. (a) TE and (b) TM reflectance spectra from $\theta=5^{\circ}$ to $65^{\circ}$ in steps of $2.5^{\circ}$ for a 1-D patterned SOI structure with lattice constant $a=1 \mu \mathrm{m}$ and 0.7 air fraction (sample L3).

\section{RESUlTS AND DisCUSSION}

The reflectance spectra of sample L3 for TE and TM polarized light incident along the $\Gamma-X$ orientation are shown in Fig. 2(a) and (b), respectively. The angle of incidence is varied from $5^{\circ}$ to $65^{\circ}$ in steps of $2.5^{\circ}$, and the spectra are shifted vertically (each one by the same quantity) in order to facilitate viewing.

The analogous reflectance spectra for sample L4 are shown in Fig. 3(a) and (b), respectively. Here the incidence angle is varied from $5^{\circ}$ to $75^{\circ}$. The corresponding calculated reflectance spectra are shown for both TE and TM polarizations in Fig. 4(a) and (b), respectively.

As a first remark, the experimental reflectance curves show a regular interference pattern resulting from the SOI waveguide structure. At first, near-normal incidence reflection spectra of the structure before patterning were well fitted by a multilayer model taking into account the waveguide structure with the actual layer thickness values. After patterning of the samples, the interference fringes are modified and change their period slightly on increasing the photon energy. This effect arises from the effective refractive index of the photonic crystal which increases with increasing energies due to the stronger confinement of the electromagnetic field in the dielectric regions with respect to the air ones. Superimposed on this interference background, several sharp features are observed which display a well-defined dispersion in their energy positions with increasing angle of incidence. While sample L3 displays rather weak and broad features, sample L4 shows more intense and well-defined structures. The resonant structures are broader in sample L3 because of the higher air fraction, which implies a larger coupling of folded guided modes above the light line to leaky waveguide modes and therefore an increase of the diffraction losses outside the waveguide. This is in agreement with recent results obtained by other groups [12], [18]. The spectra of Fig. 2 suggest that samples with large air fraction may not be useful for achieving low-loss propagation.
In the following, we perform a detailed analysis only for sample L4, whose sharp optical features point to the existence of quasi-guided modes with low losses. Comparing the reflectance curves of Figs. 3 and 4, there is a good overall agreement between experimental and calculated spectra. Both the interference pattern from the core and cladding layers and the observed resonant structures are well accounted for by calculations, although the calculated resonances appear to be slightly sharper than the experimental ones. This is probably due to some dispersion of the incidence angle which is defined within a cone of $\pm 1^{\circ}$, and possibly also to some disorder effect coming from the etching process. The intensity and the lineshape of the spectral structures (both experimental and calculated) change with the angle, showing a variety of maxima, minima, and dispersive-like lineshapes.

These resonances are due to the coupling of external radiation to guided modes of the patterned waveguide, as already reported for the GaAs-AlGaAs system [8], [9], [11], [15]. When the frequency $\omega$ and the wavevector component $\mathrm{k}$ parallel to the waveguide plane match those of a propagating mode, coupling to the waveguide structure occurs and a resonance appears in reflectance. When the angle of incidence is varied, the parallel wavevector changes and the matching condition occurs at a different energy. Therefore, the dispersion of the photonic bands in a given direction can be extracted from the energy positions of the resonances versus the wavevector $k=(\omega / c) \sin \theta$ that spans the first Brillouin zone. This analysis is shown in Fig. 5: the experimental bands derived from reflectance measurements are plotted by the dots. Since most structures in reflectance spectra show a dispersive-like shape, we take the inflection point as the energy position for the propagating mode (as indicated in Fig. 3 for $\theta=5^{\circ}$ ).

The photonic bands of the ideal 1-D crystal corresponding to the L4 structure are reported in Fig. 5(a). The photonic bands in the waveguide calculated with the method described in Section III are also reported, separated in TE (b) and TM (c) modes according to mirror symmetry with respect to the plane of incidence $(\mathbf{k}, z)$. The frequency dispersion of the dielectric constant 


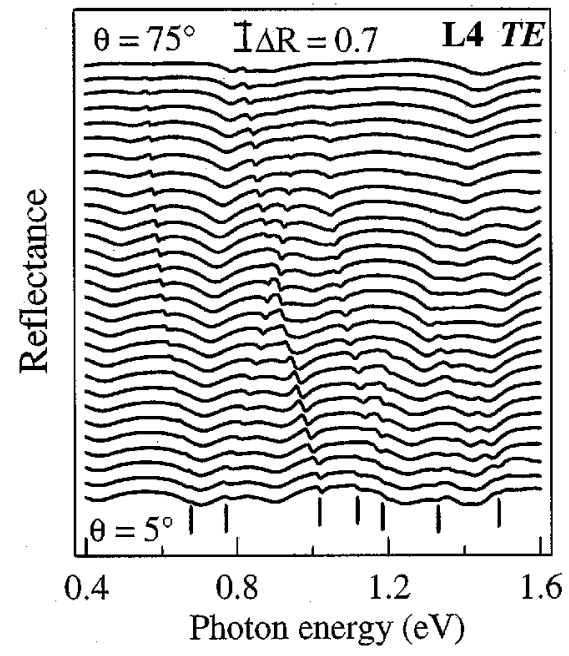

(a)

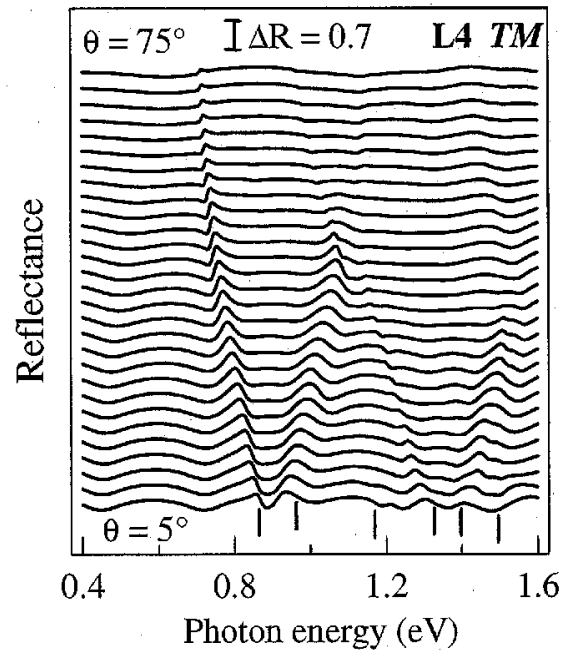

(b)

Fig. 3. (a) TE and (b) TM reflectance spectra from $\theta=5^{\circ}$ to $75^{\circ}$ in steps of $2.5^{\circ}$ for a 1-D patterned SOI structure with lattice constant $a=0.65 \mu \mathrm{m}$ and 0.18 air fraction (sample L4). Vertical bars mark the position of 1-D photonic modes for $5^{\circ}$.

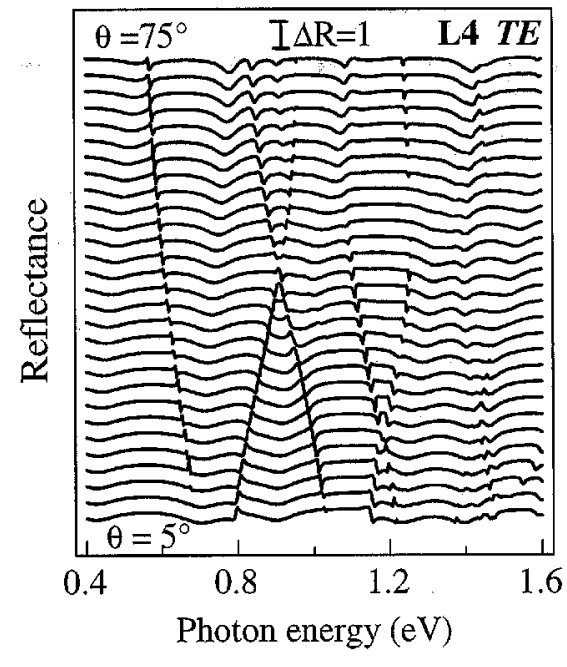

(a)

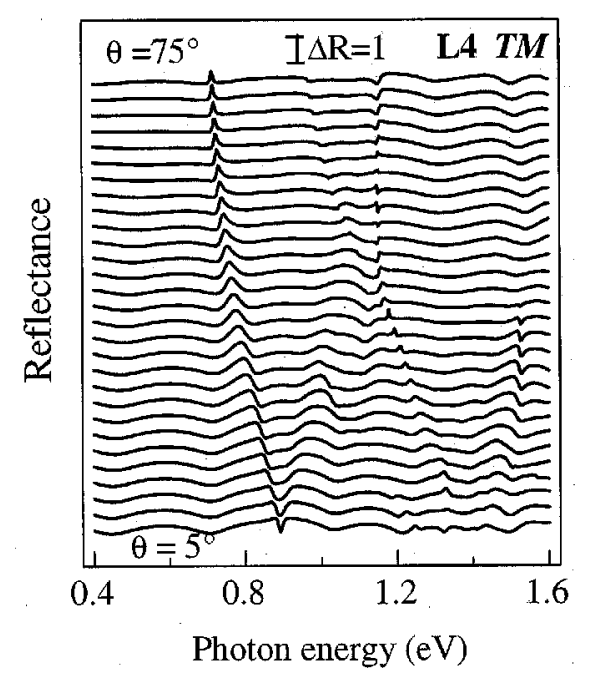

(b)

Fig. 4. (a) Calculated TE and (b) TM reflectance spectra of sample L4 from $5^{\circ}$ to $75^{\circ}$ in steps of $2.5^{\circ}$.

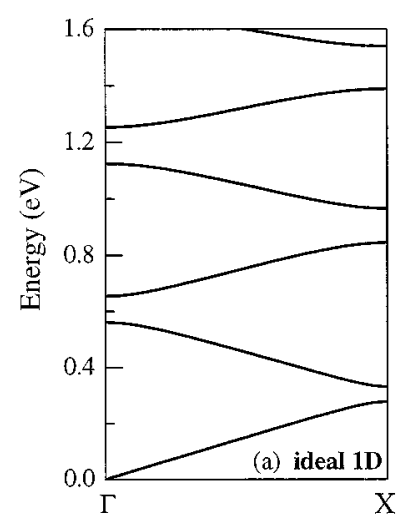

(a)

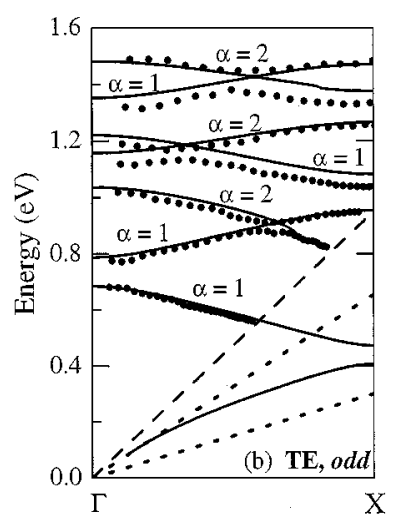

(b)

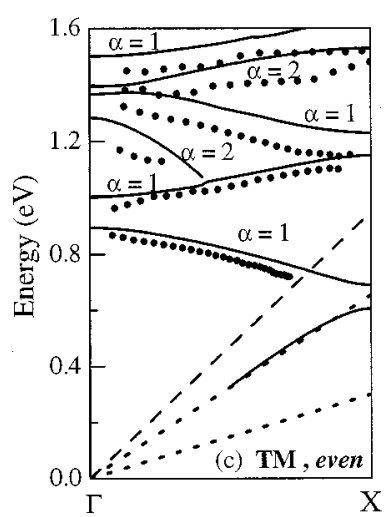

(c)

Fig. 5. Photonic bands of sample L4, calculated (solid lines) and determined from the experimental reflectance spectra (dots): (a) ideal 1-D crystal, (b) TE polarization—odd bands, and (c) TM polarization—even bands. Dotted (dashed) lines: dispersion of light in $\mathrm{Si}_{1} \mathrm{SiO}_{2}$ (in air). The index $\alpha$ in (b) and (c) refers to the order of the dominant waveguide mode (see text).

is taken into account in an approximate way by calculating each group of nearby bands with $\varepsilon(\omega)$ taken at an average frequency; the same procedure is used for the ideal 1-D and the waveguide case. Dotted lines represent the dispersion of photons in Si and 
$\mathrm{SiO}_{2}$, while dashed lines are the light lines in vacuum. The photonic bands in the waveguide are blue-shifted in energy with respect to the ideal 1-D case due to a confinement effect in the $z$ direction; the effect is stronger for the TM-polarized modes. We notice that guided modes, i.e., modes whose dispersion is confined between the $\mathrm{Si}$ core and the $\mathrm{SiO}_{2}$ dispersion lines, are present both for TE and TM polarizations: they arise from a first-order waveguide mode. However, as results from the band diagrams, the lowest band in the guided mode region has a finite cut-off wavelength imposed by the thickness of the asymmetric waveguide. The guided modes go over smoothly into the radiative region when crossing the light line and should thereafter be viewed as resonances or quasi-guided modes. The first-order waveguide mode folded in the radiative region has gaps at the Brillouin zone center and edge; a second-order waveguide mode is also seen to exist in the radiative region with a cutoff energy of about $0.8 \mathrm{eV}$ (TE polarization) and $1.1 \mathrm{eV}$ (TM polarization). The order of the waveguide modes which dominates in the expansion of (1) is indicated on the curves. Since the waveguide is asymmetric, there is no parity distinction between first- and second-order modes and their energy dispersions anticross, albeit with very small gaps.

All the calculated TE and TM bands up to $1.6 \mathrm{eV}$ are recognized in the experimental spectra. In particular, for TE bands the agreement for the dispersion and the energy values is very good, while for TM bands small discrepancies occur which increase with increasing energies. The band diagrams show that a zone-center gap for TE polarization opens around $0.75 \mathrm{eV}(1.65 \mu \mathrm{m})$ while a gap for TM polarization opens around $0.9 \mathrm{eV}(1.38 \mu \mathrm{m})$. This can be clearly observed from reflectance spectra: for TE polarization, no resonant structure beside interference oscillations is present in the energy interval $0.7-0.8 \mathrm{eV}$ for any angle of incidence. This indicates that no photonic mode is allowed in this energy range and therefore propagation through the waveguide is forbidden. A photonic bandgap in the radiative region is therefore determined in a more direct way as compared to transmission measurements, where in-plane and out-of plane diffraction as well as symmetry mismatch between the incoming and propagating mode may yield low transmission which is not associated with a bandgap. The lowest gap at the zone boundary (around $0.45 \mathrm{eV}$ for TE, around $0.65 \mathrm{eV}$ for TM polarization) is formed in the guided mode region: this portion of the $\mathbf{k}-\omega$ space cannot be accessed by surface reflectance; therefore, here one has to rely on the calculations. The very good agreement between the measured and calculated bands for higher energies indicates that the calculation of the photonic bands in the guided mode region is accurate.

\section{CONCLUSION}

Fabrication of photonic crystals on patterned SOI waveguides leads to high-quality structures with well-defined optical properties, as shown by surface reflectance which displays a number of resonant structures in correspondence with the photonic modes. Comparison of two 1-D lattices with air fractions $70 \%$ and $18 \%$ shows that the former one has much broader resonances, indicating that samples with a larger air fraction have higher diffraction losses of quasi-guided modes. The measured reflectance spectra are well reproduced by those calculated with the scattering matrix method. The photonic bands determined from the spectral positions of resonant structures agree very well with the results of an expansion into waveguide modes of the SOI structure. The present structures are promising in order to obtain a photonic bandgap around 1.3and $1.55-\mu \mathrm{m}$ wavelength. Samples with 2-D graphite lattice of pillars and triangular lattice of holes are also being fabricated and will be studied in the near future.

\section{ACKNOWLEDGMENT}

The authors acknowledge Prof. G. Guizzetti for useful suggestions and for the critical reading of the manuscript.

\section{REFERENCES}

[1] T. F. Krauss, R. M. De La Rue, and S. Brand, "Two-dimensional photonic-bandgap structures operating at near-infrared wavelengths," $\mathrm{Na}$ ture, vol. 383, pp. 699-702, 1996.

[2] S. Fan, P. R. Villeneuve, J. D. Joannopoulos, and E. F. Schubert, "High extraction efficiency of spontaneous emission from slabs of photonic crystals," Phys. Rev. Lett., vol. 78, pp. 3294-3297, 1997.

[3] D. Labilloy, H. Benisty, C. Weisbuch, T. F. Krauss, R. M. De La Rue, V. Bardinal, R. Houdré, U. Oesterle, D. Cassagne, and C. Jouanin, "Quantitative measurement of transmission, reflection, and diffraction of two-dimensional photonic band gap structures at near-infrared wavelengths,' Phys. Rev. Lett., vol. 79, pp. 4147-4150, 1997.

[4] D. Labilloy, H. Benisty, C. Weisbuch, C. J. M. Smith, T. F. Krauss, R. Houdré, and U. Oesterle, "Finely resolved transmission spectra and band structure of two-dimensinal photonic crystals using emission from InAs quantum dots," Phys. Rev. B, vol. 59, pp. 1649-1652, 1999.

[5] H. Benisty, C. Weisbuch, D. Labilloy, M. Rattier, C. J. M. Smith, T. F. Krauss, R. M. De La Rue, R. Houdré, U. Oesterle, C. Jouanin, and D. Cassagne, "Optical and confinement properties of two-dimensional photonic crystals," J. Lightwave Technol., vol. 17, pp. 2063-2077, 1999.

[6] S. G. Johnson, S. Fan, P. R. Villeneuve, J. D. Joannopoulos, and L. A. Kolodziejski, "Guided modes in photonic crystal slabs," Phys. Rev. B, vol. 60, pp. 5751-5758, 1999.

[7] T. Baba, N. Fukaya, and J. Yonekura, "Light propagation characteristics in defect waveguides in a photonic crystal slab," Electron. Lett., vol. 27, pp. 654-657, 1999.

[8] V. N. Astratov, D. M. Whittaker, I. S. Culshaw, R. M. Stevenson, M. S. Skolnick, T. F. Krauss, and R. M. De La Rue, "Photonic band-structure effects in the reflectivity of periodically patterned waveguides," Phys. Rev. B, vol. 60, pp. R16255-R16258, 1999.

[9] V. N. Astratov, I. S. Culshaw, R. M. Stevenson, D. M. Whittaker, M. S. Skolnick, T. F. Krauss, and R. M. De La Rue, "Resonant coupling of near-infrared radiation to photonic band structure waveguides," J. Lightwave Technol., vol. 17, pp. 2050-2057, 1999.

[10] D. M. Whittaker and I. S. Culshaw, "Scattering-matrix treatment of patterned multilayer photonic structures," Phys. Rev. B, vol. 60, pp. 2610-2618, 1999

[11] V. N. Astratov, R. M. Stevenson, I. S. Culshaw, D. M. Whittaker, M. S. Skolnick, T. F. Krauss, and R. M. De La Rue, "Heavy photon dispersions in photonic crystal waveguides," Appl. Phys. Lett., vol. 77, pp. 178-180, 2000.

[12] H. Benisty, D. Labilloy, C. Weisbuch, C. J. M. Smith, T. F. Krauss, D. Cassagne, A. Béraud, and C. Jouanin, "Radiation losses of waveguidebased two-dimensional photonic crystals: Positive role of the substrate," Appl. Phys. Lett., vol. 76, pp. 532-534, 2000.

[13] M. Tokushima, H. Kosaka, A. Tomita, and H. Yamada, "Lightwave propagation through a $120^{\circ}$ sharp bent single-line-defect photonic crystal waveguide," Appl. Phys. Lett., vol. 76, pp. 952-954, 2000.

[14] M. Lončar, D. Nedeljković, T. Doll, J. Vučković, A. Scherer, and T. P. Pearsall, "Waveguiding in planar photonic crystals," Appl. Phys. Lett., vol. 77, pp. 1937-1939, 2000.

[15] V. Pacradouni, W. J. Mandeville, A. R. Cowan, P. Paddon, J. F. Young, and S. R. Johnson, "Photonic band structure of dielectric membranes periodically textured in two dimensions," Phys. Rev. B, vol. 62, pp. 4204-4207, 2000. 
[16] E. Chow, S. Y. Lin, S. G. Johnson, P. R. Villeneuve, J. D. Joannopoulos, J. R. Wendt, G. A. Vawter, W. Zubrzycki, H. Hou, and A. Alleman, "Three-dimensional control of light in a two-dimensional photonic crystal slab," Nature, vol. 407, pp. 983-986, 2000.

[17] N. Kawai, K. Inoue, N. Ikeda, N. Carlsson, Y. Sugimoto, K. Asakawa, S. Yamada, and Y. Katayama, "Transmittance and time-of-flight study of $\mathrm{Al}_{x} \mathrm{Ga}_{1-x}$ As-based photonic crystal waveguides," Phys. Rev. B, vol. 63, no. 153313, 2001.

[18] N. Kawai, K. Inoue, N. Carlsson, N. Ikeda, Y. Sugimoto, K. Asakawa, and T. Takemori, "Confined band gap in an air-bridge type of two-dimensional AlGaAs photonic crystal," Phys. Rev. Lett., vol. 86, pp. 2289-2292, 2001

[19] T. Ochiai and K. Sakoda, "Dispersion relation and optical transmittance of a hexagonal photonic crystal slab," Phys. Rev. B, vol. 63, no. 125107, 2001.

[20] — "Nearly free-photon approximation for two-dimensional photonic crystal slabs," Phys. Rev. B, vol. 64, no. 045108, 2001.

[21] K. M. Ho, C. T. Chan, and C. M. Soukoulis, "Existence of a photonic gap in periodic dielectric structures," Phys. Rev. Lett., vol. 65, pp. $3152-3155,1990$.

[22] E. D. Palik, Ed., Handbook of Optical Constants of Solids. Orlando, FL: Academic, 1985.

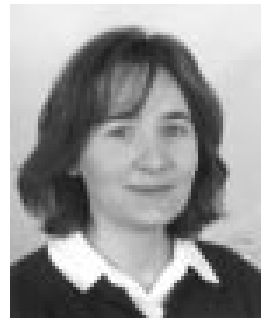

M. Patrini received the diploma and Ph.D. degrees in physics from the University of Pavia, Pavia, Italy, in 1992 and 1997, respectively.

From 1998 to 2000, she held a post-doctoral position granted by Scuola Normale Superiore in Pisa and Saint-Gobain Recherche, Italy, to perform research on clusters supported in amorphous matrix. Since 2000, she has been with the University of Pavia as a Research Scientist. Her main research interests are the optical properties of materials investigated by means of optical spectroscopies. Currently, she is involved in the experimental activity concerning the optical properties of photonic crystals.

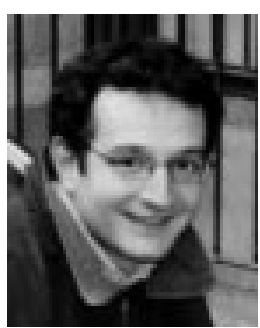

M. Galli received the diploma in physics and the Ph.D. degree from the University of Pavia, Pavia, Italy, in 1994 and 1999, respectively.

From 1999 to 2000, he held a post-doctoral position granted by the Europen Science Foundation (ESF) at the Technical University of Wien. Since 2001, he has held a post-doctoral position at the Optical Spectroscopy Laboratory at the University of Pavia. His main research activities concern strongly correlated electron systems, silicides, and, recently, spectroscopy of photonic crystals.

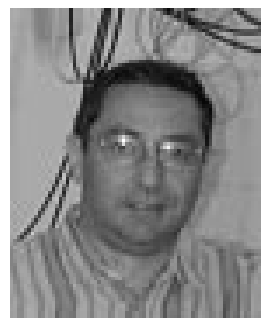

F. Marabelli was born in 1959 . He received the $\mathrm{Ph}$.D. degree from the Swiss Federal Institute of Technology (ETH) Zurich.

Ssince 1992, he has been an Associate Professor with the Science Faculty of the University of Pavia, Pavia, Italy. He is now responsible for the Optical Spectroscopy Laboratory at the Physics Department "A.Volta" of the University of Pavia. His main interest is the optical spectroscopy of solid state materials, and he has been involved in research on heavy fermions, silicides, and fullerenes. The recent topic of interest is SiGe quantum dots and photonic crystals.

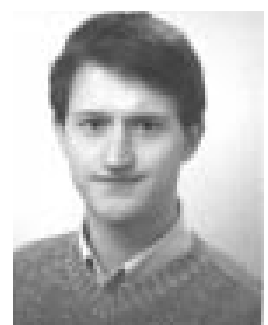

M. Agio was born in Piacenza, Italy, in 1975. He received the diploma (laurea) in physics from the University of Pavia, Pavia, Italy, in 1999. He is currently working toward the Ph.D. degree in physics at the same university and also at Iowa State University, through the Memorandum of Agreement "International Doctorate."

He was a Research Scholar at Ames Laboratory, Ames, IA, from September 1999 to December 1999. $\mathrm{He}$ is also associated with INFM and Ames Laboratory. His research activity concerns the theory of

photonic crystals.

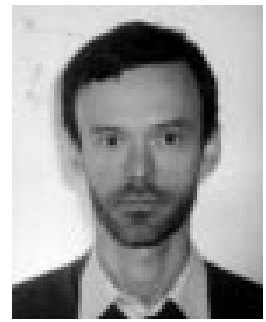

L. C. Andreani received the Ph.D. degree in physics from Scuola Normale Superiore, Pisa, Italy, in 1989. His dissertation focused on excitons and polaritons in semiconductor nanostructures.

He then was a Post-Doctoral Fellow at the IRRMA-Ecole Polytechnique Fédérale de Lausanne, Switzerland, working on strongly correlated electron systems. In 1992, he became a Researcher and in 1998 an Associate Professor at the University of Pavia, Pavia, Italy. His research interests span several areas in theoretical condensed matter physics, in particular the optical properties of semiconductors and their heterostructures. His most significant works concern excitons and radiation-matter interaction in quantum wells and in microcavities. Since 2000, he has been strongly involved in the physical investigations of photonic crystals, both from the theoretical side and in collaboration with experimentalists in Pavia and in other laboratories.

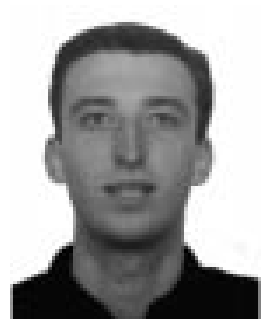

in $\mathrm{GaN}$ and SOI
D. Peyrade was born in Toulouse, France, in 1973. $\mathrm{He}$ graduated from the Physics Department of INSA and received the M.Sc. degree in physics and microelectronics from the Université Paul-Sabatier, Toulouse, in 1997. He is currently working toward the Ph.D. degree with the Groupe d'Etude des Semiconducteurs (Montpellier) and the Laboratoire de Photonique et de Nanostructures, Marcoussis, France.

His main interests are nanofabrication, optical characterization, and modeling of photonic crystals

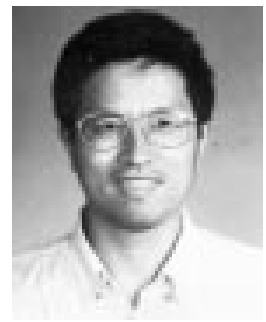

Yong Chen received the Ph.D. degree from the University of Montpellier in 1986.

$\mathrm{He}$ is a Director of Research at the Centre National de la Recherche Scientifique (CNRS), the Laboratoire de Photonique et de Nanostructures, France. Prior to joining CNRS in 1990, he worked in the Scuola Normale Superiore of Pisa and the University of Beijing between 1987 and 1990. His current interests are in the development of advanced nanofabrication technologies as well as prospective applications. He has published over 160 papers. 